\title{
A Fuzzy Action Selection Method for Virtual Agent Navigation in Unknown Virtual Environments
}

\author{
Jafreezal Jaafar; Eric McKenzie \\ Institute of Perception, Action and Behaviour, School of Informatics \\ University of Edinburgh, United Kingdom
}

Received 29 June 2007; Accepted 11 November 2007

\begin{abstract}
This paper presents an action selection method using fuzzy logic. The objective is to solve behaviour conflict in behaviour-based architectures for virtual agent navigation in unknown virtual environments. Two main problems have been identified: how to decide which behaviour should be activated at each instant; and how to combine the results from different behaviours into one action. The method uses fuzzy $\alpha$-levels to compute behaviour weight for each behaviour and the final action is selected using the Huwicz criterion. The results clearly demonstrate the mapping of inputs to output with a near-optimum path in every navigation task.

(C) 2008 World Academic Press, UK. All rights reserved.
\end{abstract}

Keywords: action selection, fuzzy logic, agent navigation, unknown Environment

\section{Introduction}

Behavior-based control is one of the three dominant paradigms for agent and robot control. Behaviours are processes or control laws that achieve and/or maintain goals. The goal of the system is achieved by subdividing the overall task into small independent behaviours that focus on execution of specific subtasks [1]. Most Behaviour-Based Systems (BBS) are reactive systems where action is executed without any planning. The action is triggered by reacting to the environment rather than deliberation or cognitive assessment.

An Action Selection Mechanism (ASM) computes which action should be executed by a BBS using the internal state and the external perceptions of the agent. Unfortunately it is difficult to make good decisions that satisfy both goal and constraint. One of the main issues is how to define the required behaviours to accomplish the goal [2]. This problem appears in the decision process when sensory data matches with several behaviour rules (conditional part of the rules), such that more than one behaviour rule is fired at the same time. As a result, behaviour rules conflict with one another, which means that more than one rule becomes active at one time.

A behaviour-based fuzzy controller and local minima algorithm has been developed in $[3,4,5]$. Therefore this paper focuses on a new fuzzy method for the action selection problem, which is based on fuzzy $\alpha$-levels incorporated with the Huwicz criterion. The objective is that the virtual agent should make an optimal decision which satisfies most of the criteria.

\section{Background}

The Action Selection Problem (ASP) is one of the fundamental problems for an autonomous system and has been studied in many fields such as ethology, artificial life, virtual reality \& graphic simulation, software agents and robotics (physical agent). For example, given a set of actions, $\bar{X}=\left\{x_{1}, x_{2}, \ldots, x_{n}\right\}$, the agent has to decide which is the most appropriate or the most relevant next action to take at a particular moment, when facing a particular situation [6].

\footnotetext{
${ }^{*}$ Corresponding author. Email: j.jaafar@sms.ed.ac.uk (J. Jaafar)
} 
Action Selection Mechanisms (ASMs) have been applied in many different areas, which present many diverse properties. No perfect ASM has been developed, since different systems have different requirements[7]. ASMs play a major role in behaviour control systems. In the decision process multiple conflicting objectives are considered simultaneously, subject to certain constraints dictated by the agent limitations [8]. A major issue in the design of systems for controlling an autonomous agent is the formulation of an effective mechanism for the combination of multiple objectives into strategies for rational and coherent behaviour [8].

In general, ASMs can be classified into two groups: arbitration and command fusion [2]. Arbitration ASMs allow one behaviour or a set of behaviours at the same time to take control for a period of time until another set of behaviours is activated. This mechanism can be one of the following:

- priority - action is selected by a central module based on a priori assigned priorities, e.g. Subsumption Architecture [9], however this has most commonly been used in reactive systems rather then behaviouralbased systems $[10]$.

- state-based - select a set of behaviours that is adequately competent to handle the situation corresponding to some given state, e.g. Temporal Sequencing [11].

- winner-take-all - action selection results from the interaction of a set of distributed behaviours that compete until one behaviour wins the competition and takes control of the robot, e.g. Activiation Network [6].

Command fusion ASMs, allow multiple behaviours to contribute to the final control of the virtual agent, which means combining recommendations from multiple behaviours to form a control action that represents their consensus. This mechanism can be divided into:

- voting - interpret the output of each behavior as votes for or against possible actions. The action with the maximum weighted sum of votes is selected, e.g. Action Voting [12].

- fuzzy - similar to voting, but uses fuzzy inferencing methods to formalize the voting approach, e.g. Fuzzy/Multivalued logic approach [2].

- superposition - combine behaviour recommendations using linear combinations, e.g. Potential Field [13].

- multi-objective - each behaviour calculates an objective function over a set of permissible actions. The action that maximizes or minimizes the objective function corresponds to the action which best satisfies the objective. Multiple behaviours are blended into a single complex behaviour that seeks to select the action that simultaneously satisfies all the objectives as closely as possible [14].

However, behaviour fusion still remains difficult with three main problems [15, 16]:

- it is hard to formulate reactive behaviours quantitatively and also there might be no applicable approach to coordinating conflict;

- competition among different reactive behaviours to achieve a good performance; and

- how to select the appropriate behaviours for robustness and efficiency in accomplishing goals.

Virtual agent navigation can occur in known and unknown environments. For a known environment, the virtual agent will have knowledge about the environment and can generate the navigation path. The methods used are based on optimization and computational intelligence, for example, as in method [17]. On the other hand in an unknown environment in which the virtual agent does not have any knowledge about the environment, a method such as sensor based control in [18] uses Adaptive Dynamic Points of Visibility (ADPV) for moving agents in dynamical unconfigured environments. Other techniques used in solving the above problems are neural networks [19], learning [20] and evolutionary algorithms [21].

An alternative solution uses fuzzy logic. The two main advantages in using a fuzzy logic approach are the ability to express partial and concurrent activations of behaviours, and the smooth transitions between behaviours [22]. Researchers such as [23] have proposed a generalised framework for a behaviour-based navigation strategy for autonomous robotics which is independent of any specific robotic development platform. [24] have used hierarchical fuzzy behaviour control for an autonomous mobile robot. [25] used a multi-layer control system for two co-operating mobile robots, which uses fuzzy logic to adapt the relative importance of a set of reactive behaviours.

\section{The Architecture}

The architecture of the fuzzy controller is comprised of three behaviours. The behaviours operate at three different ranges, with Path-Planning (PP) and Goal-Seeking (GS) behaviours in global path planning, and the Obstacle-Avoidance (OA) behaviour in local path planning as in Figure 1.

The expected features of the controller are as follows: 


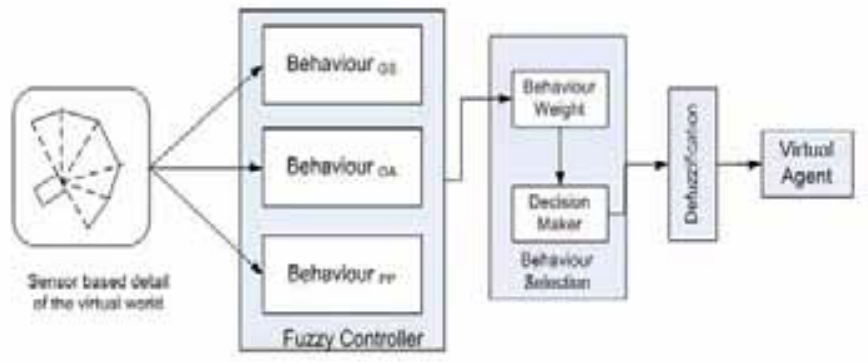

Figure 1: The Overall Architecture

- The virtual agent will make its own decisions.

- It does not require any information about the virtual environment.

- It does not require any training for the controller.

- Using fuzzy rules for the control, the system can consider operator skill and techniques.

- The control algorithm is simple. It does not require complex rules for obstacle avoidance.

The general fuzzy rules can be represented as follows:

$$
\text { IF } x \text { is } A \text { AND } y \text { is } B \text { THEN } z \text { is } C
$$

where $x, y$ and $z$ are linguistic variables for the inputs and outputs of the fuzzy controller and $A, B$ and $C$ are the terms of the variables in the universes of discourse $X, Y$ and $Z$.

A Fuzzy Associative Memory (FAM) is used as a process of encoding and mapping the input fuzzy sets to the output fuzzy set [26]. Consider a set of fuzzy rules, $R=R_{1}, R_{2}, \ldots, R_{i}, \ldots, R_{k}$, where $R_{i}$ is the $i^{\text {th }}$ rule of the fuzzy controller. The rule $R_{i}$ is given as follows:

$$
\text { IF } X_{1} \text { is } A_{1 m} \text { AND } X_{2} \text { is } A_{2 m} \text { AND ... AND } X_{n} \text { is } A_{n m} \text { THEN } Z \text { is } C_{n m}
$$

The following fuzzy relation will implement $R_{i}$ :

$$
R_{i}\left(X_{1}, X_{2}, \ldots, X_{n}, Z\right)=\left(A_{1 m} \times A_{2 m} \times \ldots \times A_{n m} \rightarrow C_{n m}\right)\left(X_{1}, X_{2}, \ldots, X_{n}, Z\right)
$$

We can rewrite equation (3) as below:

$$
R_{i}\left(X_{1}, X_{2}, \ldots, X_{n}, Z\right)=\left[A_{1 m}\left(X_{1}\right) \wedge A_{2 m}\left(X_{2}\right) \wedge \ldots \wedge A_{n m}\left(X_{n}\right)\right] \rightarrow C_{n m}(Z)
$$

Where $X_{1}, X_{2}, \ldots, X_{n}$ are input variables which are the sensor data of the virtual agent, $A_{1 m}, A_{2 m}$, $\ldots, A_{n m}$ are the input fuzzy sets, $C_{n m}$ is the output fuzzy set, $Z$ is the output variable, $n$ is the dimension of the input vector and $m$ is the number of fuzzy sets.

In order to create an $n$ fuzzy input vector $\bar{X}=\left\{\bar{X}_{10}, \bar{X}_{20}, \ldots, \bar{X}_{n 0}, \ldots, \bar{X}_{n m}\right\}$, the system needs to compose the input vector $\bar{X}$ with the calculated fuzzy relation $R_{i}$ to produce the following output $C_{i}^{\prime}$. i.e.,

$$
C_{i}^{\prime}=\left(\bar{X}_{10}, \bar{X}_{20}, \ldots, \bar{X}_{n m}\right) \circ R_{i}
$$

Where $\bar{X}_{n m}$ is the fuzzified crisp value of $X_{n m}$ into a fuzzy output class $C_{i}(Z)$. The output of the $i^{t h}$ rule $C_{i}^{\prime}(Z)$ is defined as:

$$
C_{i}^{\prime}=\left[A_{1 m}\left(X_{1}\right) \wedge A_{2 m}\left(X_{2}\right) \wedge \ldots \wedge A_{n m}\left(X_{n}\right)\right] \Rightarrow C_{i}(Z)
$$

We have used a Mamdani model and for defuzzification we use the Centroid method. The weighted sum for each individual membership can be defined as:

$$
\begin{aligned}
C & =\sum_{i=1}^{k} \omega_{i} C_{i}^{\prime} \\
& =\sum_{i=1}^{k} \omega_{i}\left(\left[A_{1 m}\left(X_{1}\right) \wedge A_{2 m}\left(X_{2}\right) \wedge \ldots \wedge A_{n m}\left(X_{n}\right)\right] \Rightarrow C_{i}(Z)\right)
\end{aligned}
$$


Where the non-negative weight $\omega_{i}$ summaries the strength of the $i^{\text {th }}$ FAM rule. The weight, $\omega$, for the $i^{\text {th }}$ FAM entry can be calculated using the minimum rule:

$$
\omega_{i}=\min \left\{C_{i}(\ell), C_{i}(\alpha)\right\}
$$

where $\ell$ is the distance between the virtual agent and an obstacle or the goal and $\alpha$ is an angle to the obstacle or the goal. Then the final defuzzification response for a $k$ output membership set is defined as:

$$
\bar{C}=\frac{\sum_{i=1}^{k} \omega_{i} C_{i}}{\sum_{i=1}^{k} \omega_{i}}
$$

Equations (4) and (9) are used to derive the FAM model and the output fuzzy system respectively. The detail of the behavioural-based fuzzy controller architecture for behaviour coordination strategy can be found in $[3]$.

\section{Visual Sensor}

The visual sensor captures the information about the virtual environment or identifies which part of an obstacle can be seen from the position of the virtual agent. Also the visual sensor only identifies whether a square (cell) in the vision range is occupied by an obstacle or not. The assumption has been made that all objects are opaque.

The visual sensor in [27] has been modified by adding a method proposed in [28] which used the DempsterShafer theory of evidence [29]. Its field of vision range is $180^{\circ}$ and five cells in distance. The vision field is divided into four main sectors which are represented as Left (L), LeftFront (LF), RightFront (RF) and Right $(R)$. Each sector provides information along a $45^{\circ}$ angle. There is a probability that the cells located in the proximity may be occupied. Cells well inside the vision field sector are likely to be empty.

The input value $\Theta$ of the virtual agent, which is a real number normalised in the interval $m: \kappa \rightarrow[0,1]$, then results from a weighted sum of all the points in the visual field.

$$
\begin{aligned}
\Theta & =\sum_{x}\left(2^{-2 d(x)} a(x)\right) \\
a(x) & =\kappa=\{0,1\}, \forall x \text { in visual field }
\end{aligned}
$$

where $d(x)$ is the distance of a point $x$ from the current position of the virtual agent, and $a(x)$ indicates the availability of the point $x$. Because the visual sensor is related to availability of spaces in the visual field, it is independent of specific environments and objects. This process will be repeated until the virtual agent reaches the goal.

\section{Action Selection Method}

The ASM method is inspired by the ranking method of Huang [30], Mabuchi [31] and Yuan [32] and uses $\alpha$-level and fuzzy subtraction operations to calculate the area of a new fuzzy number, which is produced by the comparison of two fuzzy numbers. If there are $m$ fuzzy numbers, then $m(m-1) / 2$ pairs of fuzzy numbers must be compared to determine overall rank. Our proposed method will reduce the redundancy of calculating $m(m-1) / 2$ pairwise comparisons to $m$ pairwise comparisons by the fuzzy subtraction operation.

In general, when comparing $m$ different fuzzy numbers produced by each behaviour (OA, GS, PP) the height and common maximizing and minimizing barriers are used. Let $\mu_{\widetilde{X}}(x)$ be the membership function of a fuzzy number, $\widetilde{X}$ (behaviour output), defined on $R$. Unlike convexity, $n$ assumptions about the normality of $\mu_{\widetilde{X}}(x)$ are made.

Based on [33], the loci of the left or right spreads and the maximum and minimum barriers of the $\alpha$-cut of the fuzzy number, $\widetilde{X}$, are $\mu_{\widetilde{X}_{\alpha}}^{L}(x)$ and $\mu_{\widetilde{X}_{\alpha}}^{R}(x), 0 \leq \alpha \leq h_{\widetilde{X}}$, respectively, where $h_{\widetilde{X}}$ is the height. If $\widetilde{X}_{\alpha}$ is 
denumerable or connected, then:

$$
\begin{aligned}
& \mu_{\widetilde{X}_{\alpha}}^{L}(x)=\min \left\{x \mid x \in \widetilde{X}_{\alpha}\right\}, 0 \leq \alpha \leq h_{\widetilde{X}}, \text { and } \\
& \mu_{\widetilde{X}_{\alpha}}^{R}(x)=\max \left\{x \mid x \in \widetilde{X}_{\alpha}\right\}, 0 \leq \alpha \leq h_{\widetilde{X}}
\end{aligned}
$$

The height, maximizing and minimizing barriers are set to:

$$
\begin{aligned}
h_{\widetilde{X}}(x) & =\max \left\{\mu_{X_{i}} \mid i=1,2, \ldots, m\right\}, \\
c & =\min _{\alpha}\left\{\mu_{\widetilde{X}_{\alpha}}^{L}(x) \mid i=1,2, \ldots, m ; 0 \leq \alpha \leq h_{\widetilde{X}}\right\}, \\
d & =\min _{\alpha}\left\{\mu_{\widetilde{X}_{\alpha}}^{R}(x) \mid i=1,2, \ldots, m ; 0 \leq \alpha \leq h_{\widetilde{X}}\right\} .
\end{aligned}
$$

Based on [30] and Figure 2, $h_{\widetilde{X}}(x)$ is the maximum value of the height of all $m$ fuzzy numbers. The variables

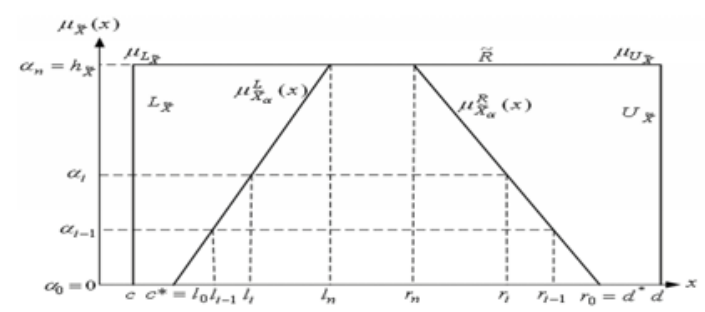

Figure 2: Trapezoidal fuzzy number

$c$ and $d$ are at the minimum value of the left spread and the minimum right spread of all fuzzy numbers, respectively. To simplify the fuzzy subtraction between the fuzzy number $\widetilde{X}$ and referential rectangle $\widetilde{R}$, at $\alpha_{i}$ level, interval subtraction is used:

$$
\begin{aligned}
\widetilde{X}_{\alpha_{i}}<->\widetilde{R} & =\left[l_{i}, r_{i}\right][-][c, d] \\
& =\left[l_{i}-d, r_{i}-c\right], i=1,2, \ldots, n
\end{aligned}
$$

then, the behaviour weight, $\omega$ of equation (13) becomes:

$$
W\left(\widetilde{X}_{i}, \widetilde{R}\right)=\frac{\sum_{i=0}^{n}\left(r_{i}-c\right)}{\sum_{i=0}^{n}\left(r_{i}-c\right)-\sum_{i=0}^{n}\left(l_{i}-d\right)}, \text { as } n=\infty
$$

where $n$ is the number of the $\alpha$-level and as $n$ approaches to $\infty$, the summation becomes the area measurement. In equation (14), $\sum_{i=0}^{n}\left(r_{i}-c\right)$ is a positive value and $\sum_{i=0}^{n}\left(l_{i}-d\right)$ is a negative value. Here, the denominator represents the total area as $n$ approaches $\infty$. In addition, if all of the aggregated fuzzy numbers are normal and within the unit interval, then $h_{i}, c=0, d=1$, and equation (14) becomes:

$$
W\left(\widetilde{X}_{i}, \widetilde{R}\right)=\frac{\sum_{i=0}^{n} r_{i}}{\sum_{i=0}^{n} r_{i}-\sum_{i=0}^{n}\left(l_{i}-1\right)} \text {, and } n=\infty
$$

In our case, the behaviour weight value $W$ from equation (15) will be used. For every $W$, we use the minimax (maximin) criterion, which selects the lowest value from each behaviour as $\delta_{1}$; and then selects the highest value from each behaviour as $\delta_{2}$. The index of optimism $\sigma[34]$, is used to represent the level of uncertainty of 
the virtual environment. When selecting one particular action from a range of possible actions, the selection is based on the Hurwicz criterion [33] which is defined as:

$$
\begin{aligned}
& \qquad \eta=\sigma \cdot\left(\min _{i=1}^{m} W_{i j_{0}}\right)+(1-\sigma) \cdot\left(\max _{i=1}^{m} W_{i j_{0}}\right) \\
& \text { where } \eta=\left\{\begin{array}{l}
\sigma=0 \rightarrow \text { maximin criterion } \\
0<\sigma<1 \rightarrow \text { compromise opinion } \\
\sigma=1 \rightarrow \operatorname{maximax} \text { criterion }
\end{array}\right.
\end{aligned}
$$

\section{Algorithm}

Based on the above discussion the following algorithm has been used. Let $\widetilde{X}_{1}, \widetilde{X}_{2}, \ldots, \widetilde{X}_{j}, \ldots, \widetilde{X}_{m}$ be $m$ arbitrary bounded fuzzy numbers produced by each behaviour.

- Step 1: Set the height $h g(x)$, common maximizing barrier $d$ and minimizing barrier $c$ for referential rectangle $\widetilde{R}$.

- Step 2: Determine the subtracted interval numbers $\left[l_{i}-d, r_{i}-c\right], i=1,2, \ldots, n$ by calculating the $n$ $\alpha$-levels for each fuzzy number $\widetilde{X}_{j}<->\widetilde{R}, j=1,2, \ldots, m$.

- Step 3: Determine the behaviour weight, $W$ for each $\tilde{X}_{j}$, by equation (15).

- Step 4: Repeat Steps 2 and 3, for every $j, j=1,2, \ldots, m$ and the $m$ behaviour weights for fuzzy numbers are obtained.

- Step 5: For every $W$, use the minimax (maximin) criterion, which selects the lowest value from each behaviour as $\delta_{1}$ and selects the highest value from each behaviour as $\delta_{2}$.

- Step 6: Determine the index of optimism $\sigma$. The final action is selected based on the Hurwicz criterion using equation (16).

\section{Evaluation}

The aim of the evaluation is to measure performance of the methods for autonomous navigation in unknown environments. The evaluation has been divided into three parts which are: basic navigation; navigate in cluttered and maze environment; and comparison with Wang [23], Fuzzy Potential Field (FPF) [35] and Fuzzy Roadmap (FRM) [36].

\subsection{Basic Navigation Skill}

The aim is to evaluate the basic skill required by a virtual agent for navigating in an unknown environment. The virtual agent is required to reach the goal avoiding the obstacles. Our experiments have demonstrated that the virtual agent has the basic skill to navigate in unknown virtual environments as in Figure 3.
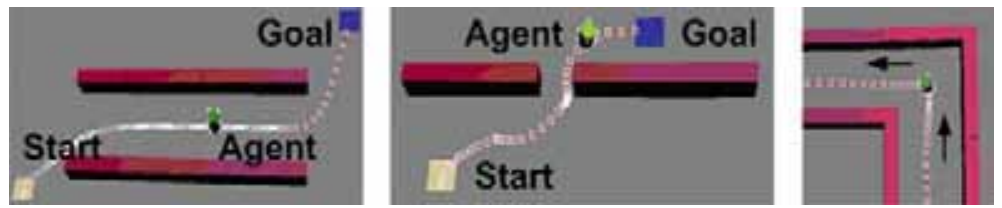

Figure 3: Basic Navigation Skill for two wall, narrow door and corner

The skills are: avoiding an obstacle; reach the goal; and navigate a narrow path (two wall, narrow door and corner). The virtual agent produced consistent results (time, number of decisions and path length) for all 25 test runs with a mean error less than $5 \%$. The path produced can be considered smooth since there is a minimum of sharp turns. For example, Figure 4 shows turn angle for the narrow door. From Table 1, the range $(\Delta \theta)$ and Mean S.E of turning angle is very small, which produces a smooth path. The result for two wall and corner shaped obstacles are very similar.

One of the major problems for local navigation is being traped in local minima. Three types of basic obstacle shape: a bench, corner and a dead-end have been used as shown in Figure 5. The simulation shows that the virtual agent avoids the obstacle, then follows the wall and heads for the target. The path generated by the virtual agent can be considered smooth even though it is not the shortest path. 


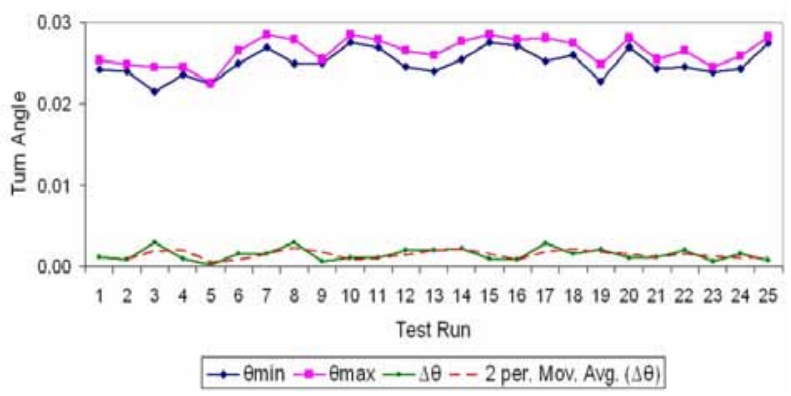

Figure 4: Turn angle for navigating through narrow door

\begin{tabular}{|c|c|c|c|}
\hline & $\theta_{\min }$ & $\theta_{\max }$ & $\Delta_{\theta}$ \\
\hline Mean & 0.025038 & 0.026493 & 0.0014541 \\
Mean S.E & 0.000340 & 0.000330 & 0.000150 \\
Std. Deviation & 0.001685 & 0.001662 & 0.000770 \\
\hline
\end{tabular}

Table 1: Statistical result for navigating through narrow door

\subsection{Navigate in cluttered and Maze Environment}

We verify the performance of our approach by applying it to cluttered and maze environments. Figure 6 shows the path produced in a cluttered environment. There are very few sharp turns and the path length can be considered short. The graph shows the degradation of Safety Index (SI) and Steering Smoothness Index (SSI) of the fuzzy controller as the degree of uncertainty increases.

The smoothness index, SSI, increases only to 1.47 times larger while the Velocity Smoothness Index (VSI) increases to 4.35 times, meaning that the VSI has more influence on the degree of uncertainty. The maximum value of SSI is $4.2^{\circ}$ and the degradation of SSI is graceful. The maximum value of VSI is 1.65 , this is relatively large compared to the maximum velocity of the virtual agent. Even if the value of uncertainty is as high as 1.0, the fuzzy controller is still able to handle the obstacle in most cases. Since the standard deviation of uncertainty measurement is not as large as $60 \%$ of the actual value in most cases, this means the fuzzy controller has high robustness to the level of uncertainty in the environment.

Figure 7 shows the path produced in a maze environment. There are some sharp turns since the virtual agent needs to make $90^{\circ}$ turns, but the other parts of the path are smooth. A similar result to that of the cluttered environment shows the degradation of Safety Index (SI) and SSI of the fuzzy controller as the degree of uncertainty increases. SSI increases only to 1.89 times while the VSI increases to 5.24 times, again meaning that the VSI has more influence on the degree of uncertainty. The maximum value of SSI is $3.5^{\circ}$, the degradation of SSI is graceful. The maximum value of VSI is 1.78, again demonstrating robustness as in the previous case.

\subsection{Comparison with Wang method [23]}

We evaluated our proposed fuzzy ASM and compared the result with the behaviour fusion method proposed by Wang in [23]. Figure 8 shows virtual agent navigation in a cluttered environment. Figure 8(a) is the path produced by our fuzzy ASM and Figure 8(b) shows the path produced by Wang's Method. The path produced by the fuzzy ASM is shorter than Wang's method even though the smoothness of the path is similar.
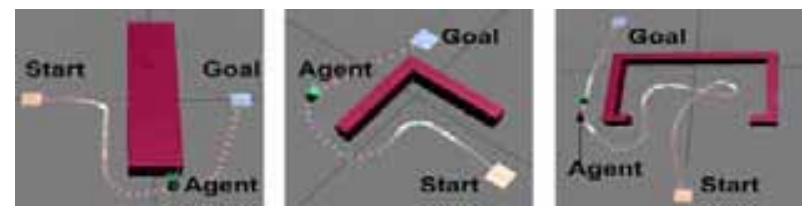

Figure 5: Escaping from bench, corner and dead-end 

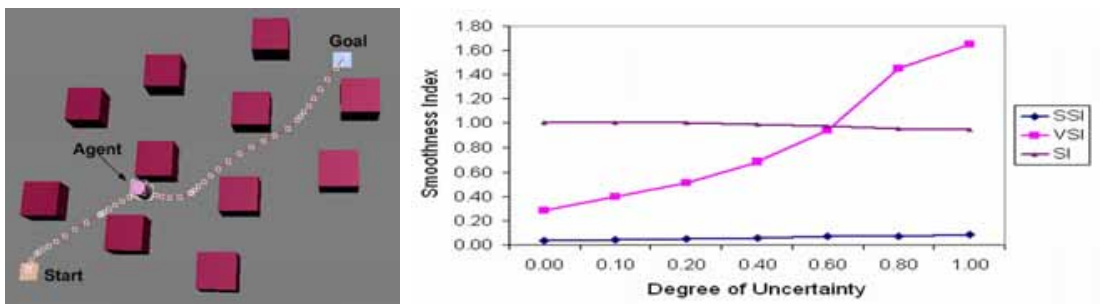

Figure 6: Smoothness vs. degree of uncertainty for cluttered environment
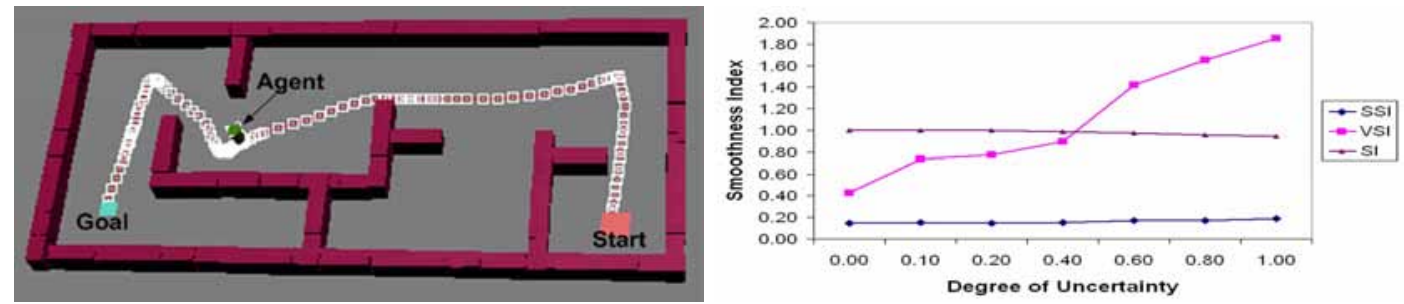

Figure 7: Smoothness vs. degree of uncertainty for maze environment.
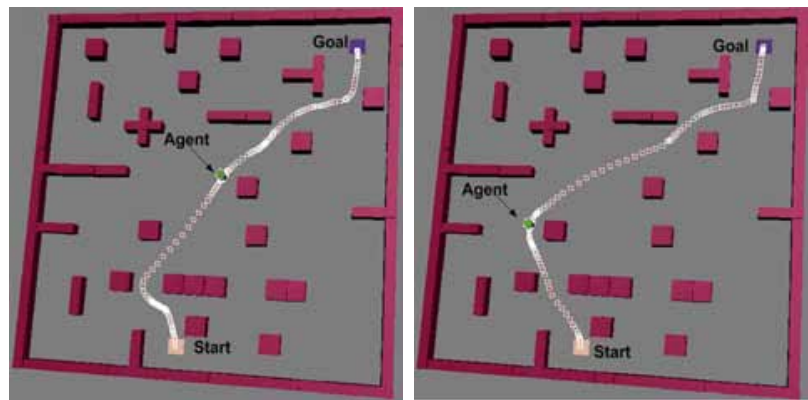

Figure 8: Path produced by (a) Fuzzy ASM and (b) Wang method 
Nine tests were conducted in nine different environments. Figure 9 show the results of time and path length taken by the virtual agent for all nine environments. The results show that the proposed fuzzy method has taken less time and shorter distance to complete the task. The average percentage of $\Delta$ time and $\Delta$ length are $16 \%$ and $17.4 \%$ respectively. When we compare the number of decisions made by each method, we see

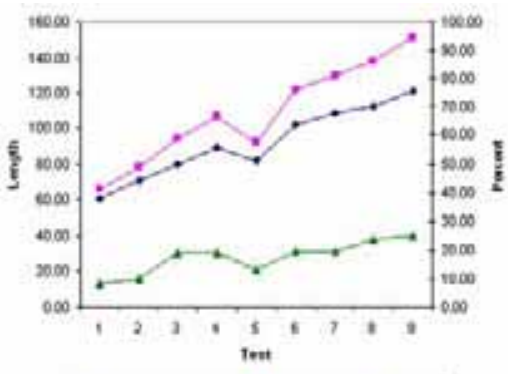

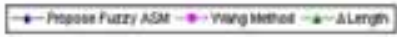

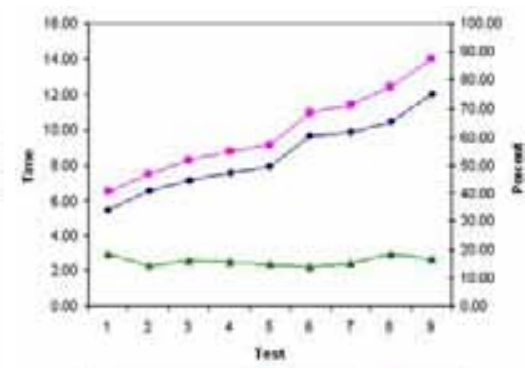

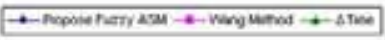

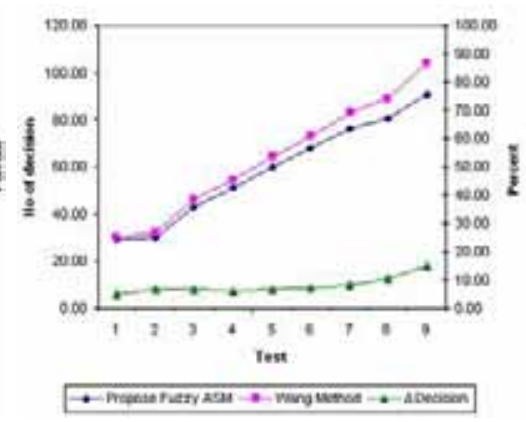

Figure 9: Fuzzy ASM vs. Wang method

that the proposed fuzzy ASM has made fewer decisions. The average number of decisions is $8.04 \%$ less than Wang's method. Fewer decisions leads to a faster and more reliable decision making process.

Our tests also show that the success rate for the fuzzy ASM is higher than Wang's method, as shown in Figure 10. Success rate refers to the percentage of test runs (total of 25 runs) for each test where the agent successfully reached the goal. In test 1 to test 4, the fuzzy ASM had a 100\% success rate. Wang's method starts to decrease at test 2 . The lowest success rate is $90 \%$ compared to Wang's method at $70 \%$. This suggests that the fuzzy ASM is more reliable.

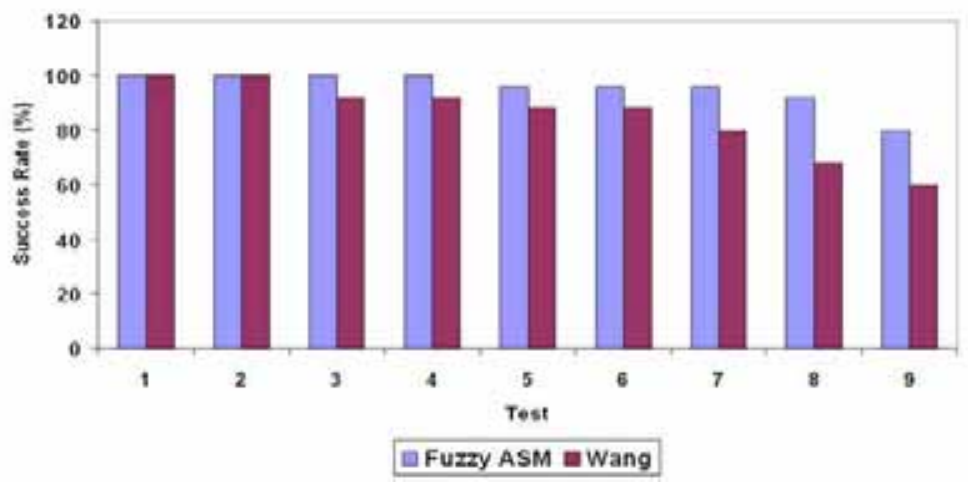

Figure 10: Comparisons of success rate

\subsection{Comparison with Fuzzy Roadmap (FRM) [36] and Fuzzy Potential Field (FPF) [35] methods}

We also compared our fuzzy ASM with other popular fuzzy methods used for agent navigation. Fuzzy Potential Field (FPF) [35] and Fuzzy Roadmap (FRM) [36] were used and a path produced by each method has been compared in Figure 11. The test results show that all methods have produced a smooth path, the main difference is the length of the path produced. Figure 12 shows the path length results for all nine tests. Our fuzzy ASM produced an average of $6.33 \%$ shorter path compared to FRM and an average of $11.59 \%$ compared to FPF, thus requiring less time to reach the goal compared to the other two methods. 

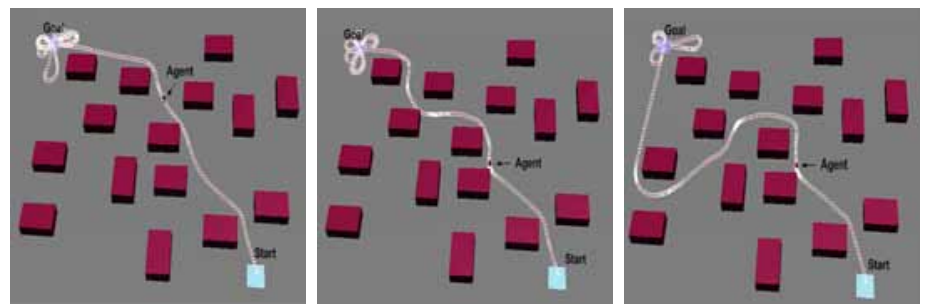

Figure 11: Example of path produced by (a) Fuzzy ASM (b) FRM (c) FPF

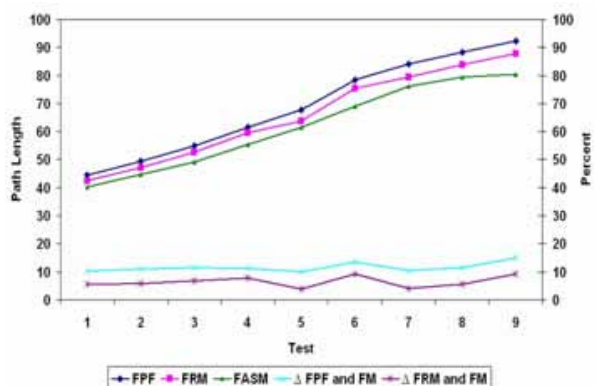

Figure 12: Path lengths produced by Fuzzy ASM, FRM and FPF

\section{Conclusion}

Our algorithm shows that behaviour rules containing $\alpha$ intervals of inputs and output spaces are easily integrated with a virtual agent. The evaluation results show that the virtual agent has deviated with minimum distance when avoiding the obstacles. The results also clearly demonstrate the mapping of inputs to output with a near-optimum path in a navigation task. This presents a natural way of dealing with a virtual environment using linguistic logic rules without having to use a complex mathematical model. The knowledge base of behaviours is easy to comprehend, because it captures the behaviour in linguistic form by a simple $\alpha$-level technique.

\section{References}

[1] Seraji, H. and A. Howard, Behavior-based robot navigation on challenging terrain: A fuzzy logic approach, IEEE Transactions on Robotics and Automation, vol.18, no.3, pp.308, 2002.

[2] Saffiotti, A., Fuzzy logic in Autonomous Robot Navigation: A case study, ser. Handbook of Fuzzy Computation, Oxford Univ. Press and IOP Press, Oxford, UK, 1998.

[3] Jaafar, J. and E. McKenzie, Behaviour coordination of virtual agent navigation using fuzzy logic, Proc. of IEEE International Conference on Fuzzy Systems, pp.1139-1145, Vancouver, Canada, 2006.

[4] Jaafar, J., E. Mckenzie and A. Smaill, A fuzzy action selection method for virtual agent navigation in unknown virtual environments, Proc. of IEEE International Conference on Fuzzy Systems, London, 2007.

[5] Jaafar, J. and E. Mckenzie, Escape from local minima for virtual agent navigation in unknown environment, Proc. of 3rd IET International Conference on Intelligent Environments, Ulm, Germany, 2007.

[6] Maes, P., How to do the right thing, Connection Science Journal, Special Issue on Hybrid Systems, 1989.

[7] Humphrys, M., Action Selection Methods Using Reinforcement Learning, Ph.D. dissertation, University of Cambridge, 1997.

[8] Pirjanian, P. and H.I. Christensen, Behavior coordination using multiple-objective decision making, Proc. of SPIE Conference on Intelligent Systems and Advanced Manufacturing, Pittsburgh, USA, 1997.

[9] Brooks, R.A., A robust layered control system for a mobile robot, IEEE Journal of Robotics and Automation, vol.RA-2, no.1, pp.14, 1986.

[10] Jones, C.V. and M.J. Mataric, Behavior-Based Coordination in Multi-Robot Systems, ser. Mobile Robots: Sensing, Control, Decision-Making, and Applications, Marcel Dekker, Inc, 2005. 
[11] Arkin, R.C. and D. MacKenzie, Temporal coordination of perceptual algorithms for mobile robot navigation, IEEE Transactions on Robotics and Automation, vol.10, no.3, pp.276, 1994.

[12] Hoff, J. and G. Bekey, An architecture for behaviour coordination learning, Proc. of IEEE International Conference on Neural Networks, vol.5, pp.2375, Perth, Australia, 1995.

[13] Khatib, O., Real-time obstacle avoidance for manipulators and mobile robots, International Journal of Robotics Research, vol.5, no.1, pp.90, 1986.

[14] Pirjanian, P. and M. Mataric, A decision-theoretic approach to fuzzy behavior coordination, Proc. of 1999 IEEE International Symposium on Computational Intelligence in Robotics and Automation, pp.101, Monterey, Canada, 1999.

[15] Li, W., Fuzzy-logic-based reactive behavior control of an autonomous mobile system in unknown environments, Engineering Applications of Artificial Intelligence, vol.7, no.5, pp.521, 1994.

[16] Perez, M.C. and J.B. Grabulosa, An overview of behavioural-based robotics with simulated implementation on an underwater vehicle, Institute of Informtica i Aplicacions, Universitat de Girona, Tech. Rep., 2000.

[17] Salomon, B, M. Garber et al., Interactive navigation in complex environments using path planning, Proc. of 2003 Symposium on Interactive 3D graphics, pp.41-50, Monterey, California, 2003.

[18] Wan, T.R., H. Chen and R. Earnshaw, Real-time path planning for navigation in unknown environment, Proc. of Theory and Practice of Computer Graphics 2003, pp.138, Birmingham, UK, 2003.

[19] Lozano, M. and J. Molina, A neural approach to an attentive navigation for 3d intelligent virtual agents, Proc. of 2002 IEEE International Conference on Systems, Man and Cybernetics, vol. 6, pp.5, Hammamet, Tunisia, 2002.

[20] Conde, T., W. Tambellini, and D. Thalmann, Behavioral animation of autonomous virtual agents helped by reinforcement learning, Proc. of 4 th International Workshop on Intelligent Virtual Agents, ser. Lecture Notes in Artificial Intelligence, vol.2792, pp.175, Springer Verlag, Germany, 2003.

[21] Gordon, V.S. and Z. Matley, Evolving sparse direction maps for maze pathfinding, Proc. of 2004 Congress on Evolutionary Computation, vol.1, pp.835, Portland, USA, 2004.

[22] Saffiotti, A., Fuzzy logic in autonomous robotics: Behavior coordination, Proc. of 6th IEEE International Conference on Fuzzy Systems, vol.1, pp.573, Barcelona, Spain, 1997.

[23] Wang, M. and J.N.K. Liu, Autonomous robot navigation using fuzzy logic controller, Proc. of 2004 International Conference on Machine Learning and Cybernetics, vol.2, pp.691, Shanghai, China, 2004.

[24] Tunstel, E., T. Lippincott, and M. Jamshidi, Behavior hierarchy for autonomous mobile robots: Fuzzy-behavior modulation and evolution, International Journal of Intelligent Automation and Soft Computing, Special Issue: Autonomous Control Engineering at NASA ACE Center, vol.3, no.1, pp.37-49, 1997.

[25] Ghanea-Hercock, R. and D.P. Barnes, Disturbed behaviour in co-operating autonomous robot, Proc. of International Conference on Autonomous Agents, pp.84, Seattle, USA, 1999.

[26] Kosko, B., Neural Networks and Fuzzy Systems: A Dynamical System Approach to Machine Intelligence, PrenticeHall inc., New Jersey, 1992.

[27] Wang, F. and E. McKenzie, A multi-agent based evolutionary artificial neural network for general navigation in unknown environments, Proc. of Third Annual Conference on Autonomous Agents, pp.154-159, Seattle, United States, 1999.

[28] Velagic, J., B. Lacevic and B. Perunicic, A 3-level autonomous mobile robot navigation system designed by using reasoning/search approaches, Robotics and Autonomous Systems, vol.54, no.12, pp.989, 2006.

[29] Shafer, G., A Mathematical Theory of Evidence, Princeton, Princeton University Press, NJ, 1976.

[30] Huang, C., A Study on The Fuzzy Ranking and its Application on The Decision Support System, Ph.D. dissertation, Tamkang University, 1989.

[31] Mabuchi, S., An approach to the comparison of fuzzy subsets with an alpha-cut dependent index, IEEE Transactions on Systems, Man and Cybernetics, vol.18, no.2, pp.264, 1988.

[32] Yuan, Y., Criteria for evaluating fuzzy ranking methods, Fuzzy Sets and Systems, vol.43, no.2, pp.139-157, 1991.

[33] Choobineh, F. and H. Li, An index for ordering fuzzy numbers, Fuzzy Sets and Systems, vol.54, no.3, pp.287, 1993.

[34] Chen, T.Y. and Y.T. Yu, On the criteria of allocating test cases under uncertainty, Proc. of Fourth Asia-Pacific Software Engineering and International Computer Science Conference, pp.405, Hong Kong, 1997.

[35] Makita, Y., M. Hagiwara and M. Nakagawa, Simple path planning system using fuzzy rules and a potential field, Proc. of IEEE International Conference on Fuzzy Systems, vol.2, pp.994, Orlando, USA, 1994.

[36] Lee, Z. and X. Chen, Path planning approach based on probabilistic roadmap for sensor based car-like robot in unknown environments, Proc. of 2004 IEEE International Conference on Systems, Man and Cybernetics, vol.3, pp.2907, The Hague, Netherlands, 2004. 